\title{
The Existence of Solutions of a Space-Uniform Boltzmann Equation
}

\author{
Zhihui Ye, Rulv Li \\ Department of Mathematics, Yunnan Nationalities University, Kunming, China \\ Email: 1139014957@qq.com
}

How to cite this paper: Ye, Z.H. and $\mathrm{Li}$, R.L. (2020) The Existence of Solutions of a Space-Uniform Boltzmann Equation. Journal of Applied Mathematics and Physics, 8 , 294-300.

https://doi.org/10.4236/jamp.2020.82023

Received: December 26, 2019

Accepted: February 9, 2020

Published: February 12, 2020

Copyright $\odot 2020$ by author(s) and Scientific Research Publishing Inc. This work is licensed under the Creative Commons Attribution International License (CC BY 4.0).

http://creativecommons.org/licenses/by/4.0/

\begin{abstract}
Boltzmann equation is an equation which is related to the three variables of $x, v, t$. In this paper, we mainly study the space-uniform Boltzmann equation which unknown function $F$ is not related to the position variable $x$. We mainly use the contraction mapping theorem to find the existence of the solution, so our mainly work is to prove the self-mapping, i.e. to prove its uniformly bounded, and then to prove the contraction mapping. There we can get the range of $\|B(\theta)\|_{\mathbf{L}^{1}\left(\mathbf{L}^{\infty}\right)}$, next we can figure out the range of $M$ and $T$ from the conditions what we know. Finally, from these conditions, we can find the existence of the solution.
\end{abstract}

\section{Keywords}

Space-Uniform Boltzmann Equations, Self-Mapping, Contractive Mapping, Uniformly Bounded, The Existence of the Solution

\section{Introduction}

Boltzmann equation is a dynamic model which describes the state of gases, and it is one of the important branches of mathematical and physical equations. It has a wide range of applications in scientific research, such as astronomy, semiconductors, aerospace technology and so on. Nowadays, many people have studied this equation, but due to its complexity and difficulty in dealing with the data, we simplify the problem most of time.

Boundary effects play an important role in the dynamics of Boltzmann solutions [1] of

$$
\partial_{t} F+v \cdot \nabla_{x} F=Q(F, F)
$$

where $F(t, x, v)$ denotes the particle distribution at time $t$, position $x \in \Omega$ and velocity $v \in \mathbb{R}^{3}$. And the collision operator takes the form 


$$
\begin{aligned}
Q(F, F) & \equiv Q_{\text {gain }}\left(F_{1}, F_{2}\right)-Q_{\text {loss }}\left(F_{1}, F_{2}\right) \\
& =\int_{\mathbb{R}^{3}} \int_{\mathbb{S}^{2}}|v-u|^{\kappa} q_{0}(\theta)\left[F_{1}\left(u^{\prime}\right) F_{2}\left(v^{\prime}\right)-F_{1}(u) F_{2}(v)\right] \mathrm{d} \omega \mathrm{d} u
\end{aligned}
$$

where $u^{\prime}=u+[(v-u) \cdot \omega] \omega, \quad v^{\prime}=u-[(v-u) \cdot \omega] \omega$ and $0 \leq \kappa \leq 1$ (hard potential) and $0 \leq q_{0}(\theta) \leq C|\cos (\theta)|$ (angular cutoff) with $\cos (\theta)=\frac{v-u}{|v-u|} \cdot \omega$.

Despite extensive developments in the study of the Boltzmann equation, many basic questions regarding solutions in a physical bounded domain, such as their regularity [2], have remained largely open. This is partly due to the characteristic nature of boundary conditions in the kinetic theory. In [3], it is shown that in convex domains, Boltzmann solutions are continuous away from the grazing set. On the other hand, in [4], it is shown that singularity (discontinuity) does occur for Boltzmann solutions in a non-convex domain, and such singularity propagates precisely along the characteristic emanating from the grazing set.

In the last years, the study of kinetic models for granular flows received a significant interest. The largest part of this work deals with kinetic nonlinear models based on generalizations of the Boltzmann-Enskog equation. Most of the studied refer to inelastic Maxwell particles, both for the driven case [5] [6] or for the free case [7] [8] [9].

In this paper, we mainly study the Boltzmann equation which unknown function $F$ is not related to the position variable $X$

$$
\left\{\begin{array}{l}
\partial_{t} F=Q(F, F) \\
F(0, v)=F_{0}
\end{array}\right.
$$

where $F(t, v) \in C\left(\mathbb{R}^{+} \times \mathbb{R}^{3}\right)$ denotes the particle distribution at time $t$ and velocity $v$. Throughout this paper, the collision operator takes the form

$$
Q(F, F)=\int_{\mathbb{R}^{3}} \int_{\mathbb{S}^{2}}|v-u|^{\kappa} q_{0}(\theta)\left[F\left(u^{\prime}\right) F\left(v^{\prime}\right)-F(u) F(v)\right] \mathrm{d} \omega \mathrm{d} u
$$

where $u^{\prime}=u+[(v-u) \cdot \omega] \omega, v^{\prime}=u-[(v-u) \cdot \omega] \omega$ and $0 \leq \kappa \leq 1$ and $0 \leq q_{0}(\theta) \leq C|\cos (\theta)|$ with $\cos (\theta)=\frac{v-u}{|v-u|} \cdot \omega$.

Theorem Assume that $F \in \mathbf{L}^{\infty}\left([0, T] \times \mathbb{R}^{3}\right)$ and for any $M$,

$$
\left\{\begin{array}{l}
M \geq \frac{1-4\|B(\theta)\|_{\mathbf{L}^{1}\left(\mathbf{L}^{\infty}\right)}\left\|F_{0}\right\|_{\mathbf{L}^{\infty}}-\sqrt{1-8\|B(\theta)\|_{\mathbf{L}^{1}\left(\mathbf{L}^{\infty}\right)}\left\|F_{0}\right\|_{\mathbf{L}^{\infty}}}}{4\|B(\theta)\|_{\mathbf{L}^{1}\left(\mathbf{L}^{\infty}\right)}} \\
M \leq \frac{1-4\|B(\theta)\|_{\mathbf{L}^{1}\left(\mathbf{L}^{\infty}\right)}\left\|F_{0}\right\|_{\mathbf{L}^{\infty}}+\sqrt{1-8\|B(\theta)\|_{\left.\mathbf{L}^{1} \mathbf{L}^{\infty}\right)}\left\|F_{0}\right\|_{\mathbf{L}^{\infty}}}}{4\|B(\theta)\|_{\mathbf{L}^{1}\left(\mathbf{L}^{\infty}\right)}}
\end{array}\right.
$$

then there exists $\|B(\theta)\|_{\mathbf{L}^{1}\left(\mathbf{L}^{\infty}\right)}$ and $T$, and they satisfy that

$$
\|B(\theta)\|_{\mathbf{L}^{1}\left(\mathbf{L}^{\infty}\right)} \leq \frac{M}{2\left(\left\|F_{0}\right\|_{\mathbf{L}^{\infty}}+M\right)^{2}}
$$




$$
T \leq \frac{\alpha}{4\|B(\theta)\|_{\mathbf{L}^{1}\left(\mathbf{L}^{\infty}\right)}\left(\left\|F_{0}\right\|_{\mathbf{L}^{\infty}}+M\right)}
$$

such that the Equation (3) exists solutions in the space $\mathbf{L}^{\infty}\left([0, T] \times \mathbb{R}^{3}\right)$.

\section{The Proof of the Result}

Define $\mathbf{X}=\left\{F \in \mathbf{L}^{\infty}\left([0, T] \times \mathbb{R}^{3}\right) \mid\left\|F-F_{0}\right\|_{\mathbf{L}^{\infty}} \leq M\right\}$, from the definition of $\mathbf{X}$, we can know that $\|F\|_{\mathbf{L}^{\infty}} \leq\left\|F_{0}\right\|_{\mathbf{L}^{\infty}}+M$.

For this equation, we need to integrate both sides of the equation with respect to $t$. Then it becomes that

$$
F=\int_{0}^{t} Q(F, F) \mathrm{d} \tau+F_{0}
$$

We denote

$$
T F=\int_{0}^{t} Q(F, F) \mathrm{d} \tau+F_{0} .
$$

Now we just need to prove that $T F=F$ is a contractive mapping.

Step 1. Prove uniformly bounded.

Before we prove that the Equation (6) is contraction mapping, we need to prove that it is uniformly bounded. So we prove $T F$ is uniformly bounded first.

By the first mean value theorem of integral, it has

$$
\left\|T F-F_{0}\right\|_{\mathbf{L}^{\infty}}=\left\|\int_{0}^{t} Q(F, F) \mathrm{d} \tau\right\|_{\mathbf{L}^{\infty}} \leq t\|Q(F, F)\|_{L^{\infty}}
$$

Next, we prove that $\|Q(F, F)\|_{L^{\infty}}$ is bounded. And we also need to prove that

$$
\|Q(F, F)\|_{L^{\infty}} \leq M
$$

So

$$
\|Q(F, F)\|_{L^{\infty}}=\sup _{0<t<t^{\prime}, v \in \mathbb{R}^{3}}\left|\int_{\mathbb{R}^{3}} \int_{\mathbb{S}^{2}}\right| v-\left.u\right|^{\kappa} q_{0}(\theta)\left[F\left(u^{\prime}\right) F\left(v^{\prime}\right)-F(u) F(v)\right] \mathrm{d} \omega \mathrm{d} u \mid(9)
$$

In the above of Equation (9), let $|v-u|^{\kappa} q_{0}(\theta)=B(\theta)$, so

$$
\begin{aligned}
\|Q(F, F)\|_{L^{\infty}} & =\sup _{0<t<t^{\prime}, v \in \mathbb{R}^{3}}\left|\int_{\mathbb{R}^{3}} \int_{\mathbb{S}^{2}} B(\theta)\left[F\left(u^{\prime}\right) F\left(v^{\prime}\right)-F(u) F(v)\right] \mathrm{d} \omega \mathrm{d} u\right| \\
& \leq \sup _{0<t<t^{\prime}, v \in \mathbb{R}^{3}} \int_{\mathbb{R}^{3}} \int_{\mathbb{S}^{2}}\left|B(\theta)\left[F\left(u^{\prime}\right) F\left(v^{\prime}\right)-F(u) F(v)\right]\right| \mathrm{d} \omega \mathrm{d} u \\
& \leq \sup _{0<t<t^{\prime}, v \in \mathbb{R}^{3}} \int_{\mathbb{R}^{3}} \int_{\mathbb{S}^{2}}\left|B(\theta) \|\left[F\left(u^{\prime}\right) F\left(v^{\prime}\right)-F(u) F(v)\right]\right| \mathrm{d} \omega \mathrm{d} u \\
& \leq \sup _{0<t<t^{\prime}, v \in \mathbb{R}^{3}} \int_{\mathbb{R}^{3}} \int_{\mathbb{S}^{2}}\left|B(\theta) \| 2 F^{2}\right| \mathrm{d} \omega \mathrm{d} u
\end{aligned}
$$

Then from Equation (8), we know $\|Q(F, F)\|_{L^{\infty}} \leq M$, thus we can get

$$
\begin{aligned}
\|Q(F, F)\|_{L^{\infty}} & \leq \sup _{0<t<t^{\prime}, v \in \mathbb{R}^{3}} \int_{\mathbb{R}^{3}} \int_{\mathbb{S}^{2}}\left|B(\theta) \| 2 F^{2}\right| \mathrm{d} \omega \mathrm{d} u \\
& \leq 2\|F\|_{\mathbf{L}^{\infty}}^{2} \int_{\mathbb{R}^{3}}\|B(\theta)\|_{\mathbf{L}^{\infty}} \mathrm{d} u \\
& \leq 2\|F\|_{\mathbf{L}^{\infty}}^{2}\|B(\theta)\|_{\mathbf{L}^{1}\left(\mathbf{L}^{\infty}\right)} \\
& \leq 2\left(\left\|F_{0}\right\|_{\mathbf{L}^{\infty}}+M\right)^{2}\|B(\theta)\|_{\mathbf{L}^{1}\left(\mathbf{L}^{\infty}\right)} \\
& \leq M
\end{aligned}
$$


From Equation (11), we can know

$$
\|B(\theta)\|_{\mathbf{L}^{1}\left(\mathbf{L}^{\infty}\right)} \leq \frac{M}{2\left(\left\|F_{0}\right\|_{L^{\infty}}+M\right)^{2}}
$$

Step 2. Prove contraction mapping.

First, we prove $T F$ is the contraction mapping, so it means we prove that

$$
\left\|T F_{1}-T F_{2}\right\|_{L^{\infty}} \leq \alpha\left\|F_{1}-F_{2}\right\|_{L^{\infty}}, 0<\alpha<1
$$

Next, we just to prove the above formula

$$
\begin{aligned}
\left\|T F_{1}-T F_{2}\right\|_{L^{\infty}}= & \sup _{|t| \leq T, v \in \mathbb{R}^{3}}\left|\int_{0}^{t}\left[Q\left(F_{1}, F_{1}\right)-Q\left(F_{2}, F_{2}\right)\right] \mathrm{d} \tau\right| \\
= & \sup _{|t| \leq T, v \in \mathbb{R}^{3}} \mid \int_{0}^{t}\left[\int _ { \mathbb { R } ^ { 3 } } \int _ { \mathbb { S } ^ { 2 } } B ( \theta ) \left(F_{1}\left(u^{\prime}\right) F_{1}\left(v^{\prime}\right)-F_{1}(u) F_{1}(v)\right.\right. \\
& \left.\left.-F_{2}\left(u^{\prime}\right) F_{2}\left(v^{\prime}\right)+F_{2}(u) F_{2}(v)\right) \mathrm{d} \omega \mathrm{d} u\right] \mathrm{d} \tau \mid \\
\leq & \sup _{|t| \leq T, v \in \mathbb{R}^{3}} \int_{0}^{t}\left[\int _ { \mathbb { R } ^ { 3 } } \int _ { \mathbb { S } ^ { 2 } } | B ( \theta ) | \left(\left|F_{1}\left(u^{\prime}\right)\right|\left|F_{1}\left(v^{\prime}\right)-F_{2}\left(v^{\prime}\right)\right|\right.\right. \\
& +\left|F_{2}\left(v^{\prime}\right)\right|\left|F_{1}\left(u^{\prime}\right)-F_{2}\left(u^{\prime}\right)\right|+\left|F_{1}(u)\right|\left|F_{1}(v)-F_{2}(v)\right| \\
& \left.\left.+\left|F_{2}(v)\right|\left|F_{1}(u)-F_{2}(u)\right|\right) \mathrm{d} \omega \mathrm{d} u\right] \mathrm{d} \tau
\end{aligned}
$$

For the above formula, we can simplify it as follows,

$$
\begin{aligned}
& \sup _{v \in \mathbb{R}^{3}}\left[\int _ { \mathbb { R } ^ { 3 } } \int _ { \mathbb { S } ^ { 2 } } | B ( \theta ) | \left(\left|F_{1}\left(u^{\prime}\right)\right|\left|F_{1}\left(v^{\prime}\right)-F_{2}\left(v^{\prime}\right)\right|+\left|F_{2}\left(v^{\prime}\right)\right|\left|F_{1}\left(u^{\prime}\right)-F_{2}\left(u^{\prime}\right)\right|\right.\right. \\
& \left.\left.+\left|F_{1}(u)\right|\left|F_{1}(v)-F_{2}(v)\right|+\left|F_{2}(v)\right|\left|F_{1}(u)-F_{2}(u)\right|\right) \mathrm{d} \omega \mathrm{d} u\right] \\
& \leq \sup _{v \in \mathbb{R}^{3}} \int_{\mathbb{R}^{3}} \int_{\mathbb{S}^{2}} 2|B(\theta)|\left(\left|F_{1}\right|+\left|F_{2}\right|\right)\left|F_{1}-F_{2}\right| \mathrm{d} \omega \mathrm{d} u \\
& \leq \sup _{v \in \mathbb{R}^{3}} \int_{\mathbb{R}^{3}} \int_{\mathbb{S}^{2}} 4|B(\theta)|\left|F \| F_{1}-F_{2}\right| \mathrm{d} \omega \mathrm{d} u \\
& \leq 4\|F\|_{L^{\infty}}\left\|F_{1}-F_{2}\right\|_{L^{\infty}} \int_{\mathbb{R}^{3}}\|B(\theta)\|_{L^{\infty}} \mathrm{d} u \\
& =4\|B(\theta)\|_{L^{1}\left(\mathbf{L}^{\infty}\right)}\|F\|_{L^{\infty}}\left\|F_{1}-F_{2}\right\|_{L^{\infty}}
\end{aligned}
$$

There, we substituted Equation (15) into Equation (14), and we can get

$$
\begin{aligned}
\left\|T F_{1}-T F_{2}\right\|_{\mathbf{L}^{\infty}} & =\sup _{|t| \leq T, v \in \mathbb{R}^{3}}\left|\int_{0}^{t}\left[Q\left(F_{1}, F_{1}\right)-Q\left(F_{2}, F_{2}\right)\right] \mathrm{d} \tau\right| \\
& \leq \sup _{|t| \leq T, v \in \mathbb{R}^{3}} \int_{0}^{t} 4\|B(\theta)\|_{\mathbf{L}^{1}\left(\mathbf{L}^{\infty}\right)}\|F\|_{\mathbf{L}^{\infty}}\left\|F_{1}-F_{2}\right\|_{\mathbf{L}^{\infty}} \mathrm{d} \tau \\
& =\sup _{|t| \leq T, v \in \mathbb{R}^{3}} \int_{0}^{t} 4\|B(\theta)\|_{\mathbf{L}^{1}\left(\mathbf{L}^{\infty}\right)}\left(\left\|F_{0}\right\|_{\mathbf{L}^{\infty}}+M\right)\left\|F_{1}-F_{2}\right\|_{\mathbf{L}^{\infty}} \mathrm{d} \tau
\end{aligned}
$$

And then from Equation (13) and the first mean value theorem, we can get Equation (16) becomes as follows

$$
\begin{aligned}
\left\|T F_{1}-T F_{2}\right\|_{L^{\infty}} & =\sup _{|t| \leq T, v \in \mathbb{R}^{3}}\left|\int_{0}^{t}\left[Q\left(F_{1}, F_{1}\right)-Q\left(F_{2}, F_{2}\right)\right] \mathrm{d} \tau\right| \\
& \leq \sup _{|t| \leq T, v \in \mathbb{R}^{3}} \int_{0}^{t} 4\|B(\theta)\|_{\mathbf{L}^{1}\left(\mathbf{L}^{\infty}\right)}\left(\left\|F_{0}\right\|_{\mathbf{L}^{\infty}}+M\right)\left\|F_{1}-F_{2}\right\|_{\mathbf{L}^{\infty}} \mathrm{d} \tau \\
& \leq 4 T\|B(\theta)\|_{\mathbf{L}^{1}\left(\mathbf{L}^{\infty}\right)}\left(\left\|F_{0}\right\|_{\mathbf{L}^{\infty}}+M\right)\left\|F_{1}-F_{2}\right\|_{\mathbf{L}^{\infty}} \\
& \leq \alpha\left\|F_{1}-F_{2}\right\|_{\mathbf{L}^{\infty}}
\end{aligned}
$$


Then, we need to prove that

$$
4 T\|B(\theta)\|_{\mathbf{L}_{1}\left(\mathbf{L}^{\infty}\right)}\left(\left\|F_{0}\right\|_{\mathbf{L}^{\infty}}+M\right) \leq \alpha
$$

From Equation (18), we can get that there exists $T$ such that the following formula holds.

$$
\|B(\theta)\|_{\mathbf{L}_{1}\left(\mathbf{L}^{\infty}\right)} \leq \frac{\alpha}{4 T\left(\left\|F_{0}\right\|_{\mathbf{L}^{\infty}}+M\right)}
$$

At last, to ensure $T F$ is a contractive mapping, so we need to find the $\|B(\theta)\|_{L^{\infty}}$ satisfy the following formula at the same time

$$
\left\{\begin{array}{l}
2\|B(\theta)\|_{\mathbf{L}^{1}\left(\mathbf{L}^{\infty}\right)}\left(\left\|F_{0}\right\|_{\mathbf{L}^{\infty}}+M\right)^{2} \leq M \\
4 T\|B(\theta)\|_{\mathbf{L}^{1}\left(\mathbf{L}^{\infty}\right)}\left(\left\|F_{0}\right\|_{\mathbf{L}^{\infty}}+M\right) \leq \alpha \\
q_{0}(\theta) \leq C|\cos (\theta)|
\end{array}\right.
$$

with $C$ is a constant.

So from Equation (12) and Equation (19), we have

$$
\|B(\theta)\|_{\mathbf{L}^{1}\left(\mathbf{L}^{\infty}\right)} \leq \min \left\{\frac{M}{2\left(\left\|F_{0}\right\|_{\mathbf{L}^{\infty}}+M\right)^{2}}, \frac{\alpha}{4 T\left(\left\|F_{0}\right\|_{\mathbf{L}^{\infty}}+M\right)}\right\}
$$

as long as the above formula holds, then the Equation (3) has solutions in the space $\mathbf{L}^{\infty}\left([0, T] \times \mathbb{R}^{3}\right)$.

In addition, from Equation (12), we can get a one-variable quadratic inequality about $M$.

$$
2\|B(\theta)\|_{\mathbf{L}^{1}\left(\mathbf{L}^{\infty}\right)} M^{2}+\left(4\|B(\theta)\|_{\mathbf{L}^{1}\left(\mathbf{L}^{\infty}\right)}\left\|F_{0}\right\|_{\mathbf{L}^{\infty}}-1\right) M+2\|B(\theta)\|_{\mathbf{L}^{1}\left(\mathbf{L}^{\infty}\right)}\left\|F_{0}\right\|_{\mathbf{L}^{\infty}}^{2} \leq 0
$$

According to this inequality, the discriminant of this inequality is more than or equal to 0 . So

$$
\left(4\|B(\theta)\|_{\mathbf{L}^{1}\left(\mathbf{L}^{\infty}\right)}\left\|F_{0}\right\|_{\mathbf{L}^{\infty}}-1\right)^{2}-4 \times 2\|B(\theta)\|_{\mathbf{L}^{1}\left(\mathbf{L}^{\infty}\right)} \times 2\|B(\theta)\|_{\mathbf{L}^{1}\left(\mathbf{L}^{\infty}\right)}\left\|F_{0}\right\|_{\mathbf{L}^{\infty}}^{2} \geq 0
$$

then, simplify the upper form, we have

$$
8\|B(\theta)\|_{\mathbf{L}^{1}\left(\mathbf{L}^{\infty}\right)}\left\|F_{0}\right\|_{\mathbf{L}^{\infty}} \leq 1
$$

So, we can get the value range of $M$.

$$
\left\{\begin{array}{l}
M \geq \frac{1-4\|B(\theta)\|_{\mathbf{L}^{1}\left(\mathbf{L}^{\infty}\right)}\left\|F_{0}\right\|_{\mathbf{L}^{\infty}}-\sqrt{1-8\|B(\theta)\|_{\mathbf{L}^{1}\left(\mathbf{L}^{\infty}\right)}\left\|F_{0}\right\|_{\mathbf{L}^{\infty}}}}{4\|B(\theta)\|_{\left.\mathbf{L}^{1} \mathbf{L}^{\infty}\right)}} \\
M \leq \frac{1-4\|B(\theta)\|_{\mathbf{L}^{1}\left(\mathbf{L}^{\infty}\right)}\left\|F_{0}\right\|_{\mathbf{L}^{\infty}}+\sqrt{1-8\|B(\theta)\|_{\mathbf{L}^{1}\left(\mathbf{L}^{\infty}\right)}\left\|F_{0}\right\|_{\mathbf{L}^{\infty}}}}{4\|B(\theta)\|_{\mathbf{L}^{1}\left(\mathbf{L}^{\infty}\right)}}
\end{array}\right.
$$

From the upper conclusion and with Equation (19), we can get the range of $T$ 


$$
T \leq \frac{\alpha}{4\|B(\theta)\|_{\mathbf{L}^{1}\left(\mathbf{L}^{\infty}\right)}\left(\left\|F_{0}\right\|_{\mathbf{L}^{\infty}}+M\right)} .
$$

\section{Conclusion}

In this paper, we mainly discussed the Boltzmann equation which is independent of the position relation $x$, and the equation independent of $x$ can be regarded as a squeezed fixed point mapping. So our main work is to prove the compressed mapping, and before we prove the compressed mapping, we need to ensure that the $T F$ we want to prove is self-mapping, that is, to prove that its uniformly bounded. In this process, we find out a relation to make the proof valid, and then in the process of proving the compressed mapping, we find out another condition to make the proof valid. Finally, we must ensure that the two conditions found earlier and the conditions required in the equation are satisfied at the same time. We mainly prove the existence of the equation by means of the compression mapping of the function.

\section{Supported}

The work is supported by National Natural Science Foundation of China (No. 11861078).

The work is supported by National Natural Science Foundation of China (No. 11561076).

\section{Conflicts of Interest}

The authors declare no conflicts of interest regarding the publication of this paper.

\section{References}

[1] Guo, Y., Kim, C., Tonon, D., et al. (2017) Regularity of the Boltzmann Equation in Convex Domains. Inventiones Mathematicae, 207, 115-290. https://doi.org/10.1007/s00222-016-0670-8

[2] Guo, Y. (1994) Regularity for the Vlasov Equations in a Half Space. Indiana University Mathematics Journal, 43, 255-321. https://doi.org/10.1512/iumj.1994.43.43013

[3] Guo, Y. (2008) Decay and Continuity of Boltzmann Equation in Bounded Domains.

[4] Kim, C. (2011) Formation and Propagation of Discontinuity for Boltzmann Equation in Non-Convex Domains. Communications in Mathematical Physics, 308, 641-701. https://doi.org/10.1007/s00220-011-1355-1

[5] Bobylev, A.V. and Cercignani, C. (2002) Moment Equations for a Granular Material in a Thermal Bath. Journal of Statistical Physics, 106, 547-567. https://doi.org/10.1023/A:1013754205008

[6] Carrillo, J., Cercignani, C. and Gamba, I. (2000) Steady States of a Boltzmann Equation for Driven Granular Media. Physical Review E: Covering Statistical, Nonlinear, Biological, and Soft Matter Physics, 62, 7700-7707. https://doi.org/10.1103/PhysRevE.62.7700

[7] Bobylev, A.V., Carrillo, J.A. and Gamba, I.M. (2000) On Some Properties of Kinetic 
and Hydrodynamic Equations for Inelastic Interactions. Journal of Statistical Physics, 98, 1137-1138. https://doi.org/10.1023/A:1010325409175

[8] Bobylev, A.V., Cercignani, C. and Toscani, G. (2003) Proof of an Asymptotic Property of Self-Similar Solutions of the Boltzmann Equation for Granular Materials. Journal of Statistical Physics, 111, 403-417.

https://doi.org/10.1023/A:1022273528296

[9] Bobylev, A.V. and Cercignani, C. (2003) Self-Similar Asymptotics for the Boltzmann Equation with Inelastic and Elastic Interactions. Journal of Statistical Physics, 110, 333-375. https://doi.org/10.1023/A:1021031031038 